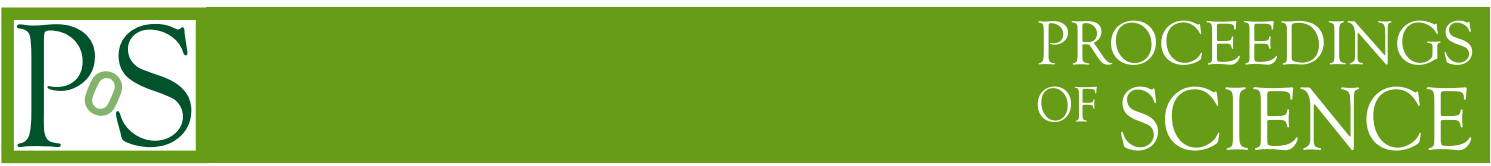

\title{
Non-Gamma-ray Applications of TeV Telescopes
}

\author{
Michael Daniel* \\ Center for Astrophysics | Harvard \& Smithsonian \\ E-mail: michael.daniel@cfa.harvard.edu
}

Imaging Atmospheric Cherenkov Telescopes (IACTs) are instruments for the indirect detection of very high energy cosmic and gamma rays, since they are essentially very large light buckets used to gather the optical Cherenkov photons generated within extensive air showers. Whilst the imaging quality of their reflective surfaces may be crude it should not be forgotten that they are in actuality amongst the largest optical telescopes ever constructed - with sensitive camera equipment ideal for application to high time resolution optical astronomical research from scales of seconds down to nanoseconds. Recent advances in instrumentation have resulted in the VERITAS IACTs making sub-milliarcsecond resolution observations through the revived technique of intensity interferometry and obtaining the smallest optical angular resolution measurements to date in direct measurements of stellar diameters at the $\sim 100$ micro-arcsecond scale from observations of asteroid occultations of stars. There are many further diverse non-gamma-ray applications that these types of telescopes are starting to contribute to: from the simultaneous search for optical counterparts to Fast Radio Bursts, to the search for extraterrestrial intelligence. This increased range of utility for these form of telescope expands their potential remit from facilities for specialised purposes to observatories for a wider scientific community in prime locations around the world.

36th International Cosmic Ray Conference -ICRC2019-

July 24th - August 1st, 2019

Madison, WI, U.S.A.

${ }^{*}$ Speaker. 


\section{Introduction}

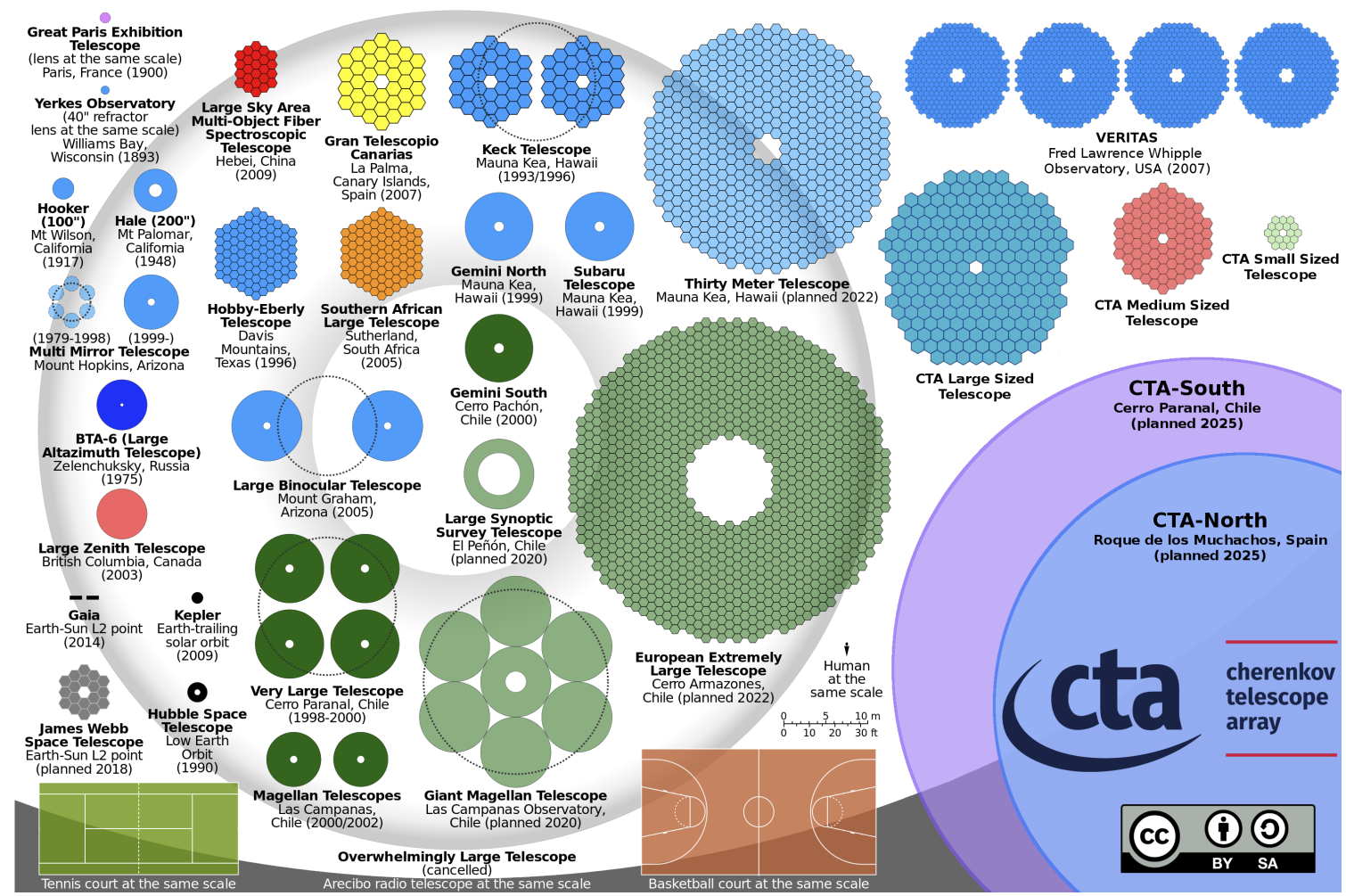

Figure 1: Comparison of the primary mirror area of the different segmented facets of past, current and future optical telescopes, including VERITAS and CTA telescopes. Figure adapted by T. Hassan from [1].

In lists of extremely large telescopes Cherenkov telescopes are often neglected but, as can be seen in Figure 1, these simple light buckets measure up with the biggest and best of them in terms of light collecting power [2]. Though the optical surface of a Cherenkov telescope is modest at best in terms of imaging performance this is not actually that much of a constraining factor when it comes to investigations of high time resolution photometry. The noise budget in photometry can be broken down to three main components: $\sigma_{n}^{2}=\sigma_{f}^{2}+\sigma_{s}^{2}+\sigma_{P}^{2}$, where $\sigma_{f}$ is the flicker noise of the observed source, $\sigma_{P}^{2}$ is the combined Shot noise of the light sources in the field of view, and $\sigma_{s}$ is the scintillation noise induced by the optical path through the atmosphere. Each of these is a complex superposition of physical processes that can really only be measured empirically, but a number of generalities can be made. For a steady point-like source scintillation will be the dominant noise component. There are many prescriptions for estimating scintillation noise, of which a few are plotted in Figure 2, but all are in agreement that for spatial averaging to reduce this noise component the bigger the aperture the better, and large apertures is something that the telescopes used for ground based TeV studies excel in. Scintillation acts as a lens in the high atmosphere and focuses light on or off the telescope mirror surface - the larger the aperture the less likely the light is to be lost by going over the mirror's edge. The next way to reduce scintillation noise is by temporally averaging as the speckle pattern moves in an out of the pixel field of view. This understanding then gives three regimes as a function of exposure time for scintillation noise [3]: for long exposures 


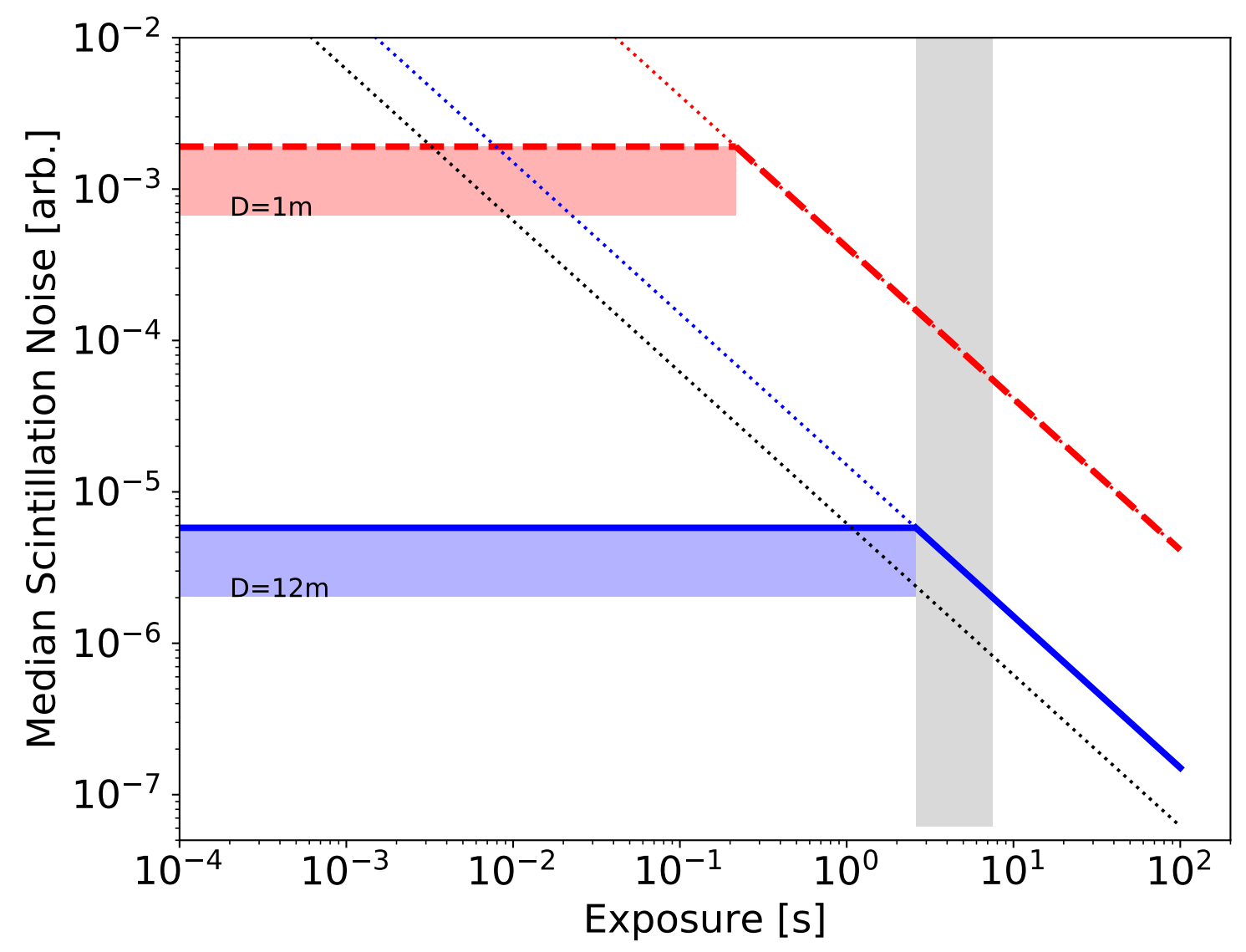

Figure 2: The scale of noise from atmospheric scintillation as a function of exposure time for a telescope of $1 \mathrm{~m}$ (red dashed) and $12 \mathrm{~m}$ (blue solid line) aperture following the scheme of [3]. For reference are plotted Young's empirical approximation (black dotted line) [4], often used by astronomers to estimate scintillation noise budget, but tends to underestimate the contribution by up to $50 \%$ and that for small telescopes in [5] (blue and red dotted lines) which again could lead to an overestimation if the site dependent scintillation speckle size which determine when temporal averaging "freezes" the magnitude of the scintillation noise component. The shaded areas show the magnitude of the uncertainty in the scintillation component depending on whether the wind velocity is parallel or perpendicular to the observed line of sight.

the speckles traverse the field of view and can be reduced by temporal averaging, the exact amount being dependent on the wind speed; for short exposures the intensity fluctuations appear frozen and no temporal averaging occurs; and for small telescopes of approximately the same size as the speckles and smaller (of order $\leq 1 \mathrm{~m}$ ) there will be a significant wavelength dependence as the size of the speckles is wavelength dependent. The large mirror surface of a Cherenkov telescope then has the twin advantages of alleviating this wavelength dependence and increasing the exposure time phase space where the scintillation noise is approximately "frozen". For one order of magnitude increase in telescope aperture size, two orders of magnitude improvement in signal to noise can be easily expected, and given the modest optics of a Cherenkov telescope at a significant saving in terms of cost, too. 


\subsection{An historical perspective}

The capabilities of Cherenkov and fluorescence telescopes to be used in optical studies has certainly been recognised in the past: the Whipple $10 \mathrm{~m}$ telescope produced the first measurements of meteor fluxes down to photographic magnitudes of +12 [6] in the 1970's and limits on the nuclearite dark matter candidate particles [7, 8] which would have a similar signature to meteors, but travel at Galactic rather than solar system velocities have been made in the 1980's on the back of the knowledge gained. Optical observations can either make use of the existing focal plane instrumentation infrastructure, e.g. $[9,10,11,21]$, or be externally mounted on to the camera housing, as in $[12,13]$. Most uses of Cherenkov telescopes in the optical regime, however, have concentrated on measurements of the Crab optical pulsar signal either to calibrate the timing systems of these instruments for $\gamma$-ray pulsar searches [14, 15, 16, 17, 18, 19, 20, 21, 13]. More speculative searches for microsecond flaring in stars [12] or for evidence of interstellar laser communications in OSETI $[47,48,49]$ have only turned up background signals due to meteors, satellites and terrestrial space debris. Whilst those are worthy scientific studies in their own right, the Crab studies have stopped short of fully exploiting the available data and the searches into the unknown have neglected targeting something with an a priori predictable outcome. Whilst less glamorous there can still be truly remarkable results achieved when observing a known optical phenomenon.

\section{Eclipses, Occultations, and Transits}

In a direct coupled system the amount of current output by a photomultiplier directly correlates to the intensity of the light it receives. Whilst the absolute intensity may not be very accurately known, any relative change in light level can still be very precisely measured. It is difficult to predict when, or whether, an astronomical source will flare up, but when one astronomical object passes in front of another it casts a shadow - such as in an eclipsing binary system, a transiting exoplanet, or an asteroid occultation - making for a very predictable change in light level that can be easily observed with the appropriate equipment. Figure 3 shows the lightcurve as a function of orbital phase for the eclipsing binary system Z Vul which consists of a B3/5 star and an A3IV star in a $\sim 2.5$ day orbit. Due to the differing star sizes, the primary transit results in a $75 \%$, and the secondary transit a $25 \%$, drop in the $7.40 \mathrm{~B}$ magnitude flux from the system. The VERITAS DC Current Monitor [9] measures the camera pixel currents to $0.5 \mu \mathrm{A}$ resolution up to $120 \mu \mathrm{A}$, at the magnitude of $\mathrm{ZVul}$ this monitoring system should be sensitive to changes in light level down to $3 \%$ and so should be clearly able to resolve both the primary and secondary eclipses. Indeed, once the observational considerations due to changing airmass, NSB, day-to-day weather variations, etc are taken into account the orbital lightcurve of this binary system is quite well reconstructed even with such a relatively crude secondary monitoring instrument of a standard Cherenkov camera.

The shadow cast contains a lot of information about the occulting object: for instance, the transit time giving a chord of an asteroid's radius, or the transit depth giving the ratio of an exoplanet's radius to that of the host star. When the astronomical object casting the shadow is within a few astronomical units of the observer they will be within the Fresnel diffraction pattern zone of the shadow and this enables us to directly measure the spatial extension of the stellar light source $[22,23,24]$. In figure 4 is plotted the lightcurve of the observation of the asteroid (1165) Imprinetta 


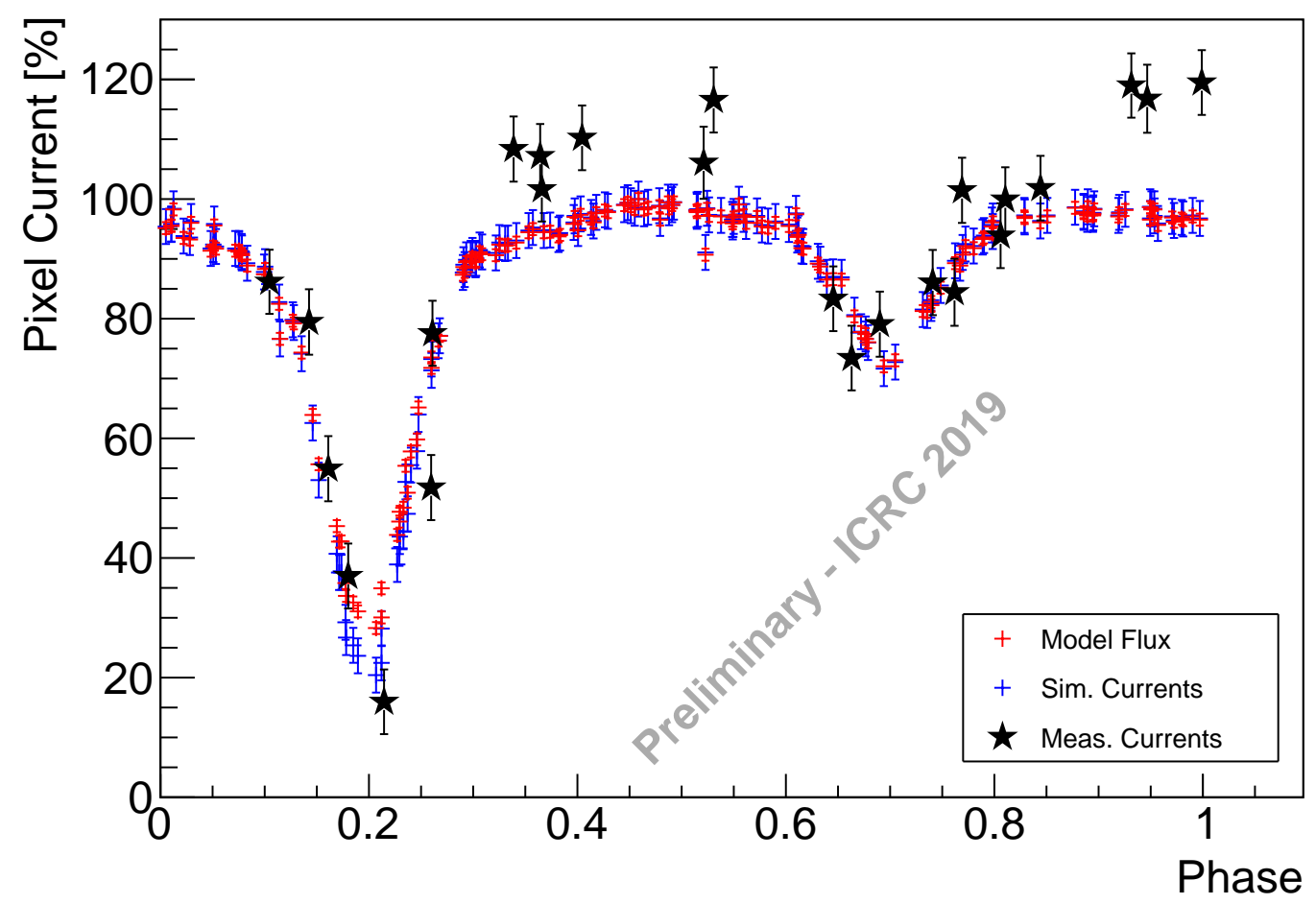

Figure 3: The orbital lightcurve of the Z Vul eclipsing binary system. The red crosses give the expected change in intensity based on prior optical telescope observations from the ASAS Catalogue of Variable Stars [25]. The blue crosses give the simulated currents expected for a system of the same visual magnitude. The black stars give the airmass corrected measurements from the VERITAS focal plane pixel current monitoring system of this system.

occulting the star TYC 5517-227-1 taken with an enhanced current monitor on the central pixel of one of the VERITAS telescopes. Even though the relative 100\% drop in starlight intensity from the occultation is nominally in excess of that for the eclipsing binary, the much smaller initial intensity of the 10th magnitude star being observed ( $\sim 2$ visual magnitudes below the night sky background contribution to the pixel current) means the signal of the occultation is at/below the resolution of the standard VERITAS focal plane current monitoring system [9], but it is well within the capabilities of even an off-the-shelf datalogger like the DATAQ D-710-ELS used in the enhancement to detect both the occultation and the fringes of the diffracted starlight (which cause a maximum intensity brightening of $40 \%$ of the starlight in the first diffraction fringe for a point-like source). The deviation of the fringe profile from that expected for a point-like source enabled these Cherenkov telescopes to directly measure the angular diameter of stars to $\leq 0.1$ mas) $[10,26]$ for the highest angular resolution measurements made in the optical regime (visual wavebands) to date (and at a level $\sim 10^{6}$ orders of magnitude smaller than the IACT design point spread function $\left(\approx 0.1^{\circ}\right)$.

There are several aspects that can constrain the achievable limit on angular resolution $\left(\phi_{m}\right)$ from observing an occultation in the Fresnel diffraction approximation region [23]:

$$
\phi_{m} \simeq \sqrt{\Delta \lambda / D}
$$



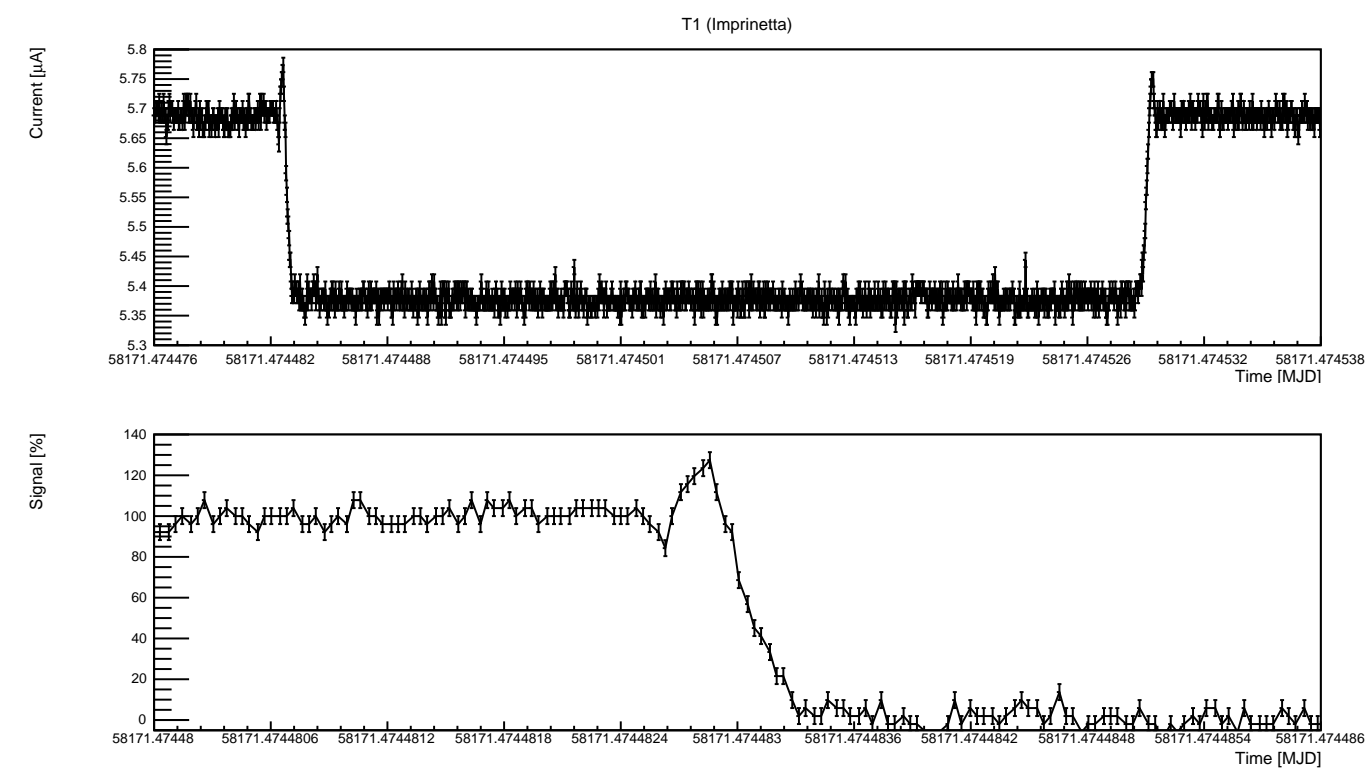

Figure 4: Top: The light curve of the ingress (left) and egress (right) of the $\sim 4 \mathrm{~s}$ (1165) Imprinetta / TYC 5517-227-1 occultation [10] taken with the VERITAS T1 enhanced current monitor with $300 \mathrm{~Hz}$ sampling rate. Bottom: A close up of the ingress portion of the lightcurve, the first diffraction fringe causes a $<40 \%$ variation of the starlight signal.

$$
\begin{aligned}
& \phi_{m} \simeq v_{f} / D f_{m} \\
& \phi_{m} \simeq 2 d / D
\end{aligned}
$$

where $D$ is the distance between the observer and the occulting object, $d$ the telescope aperture, $\Delta \lambda$ the optical bandwidth of the detector, and $v_{f}$ the shadow ground velocity. It is clear to see that the power of asteroid occultation, over say lunar occultation, comes in the large distance to the occulting object. The downside for this technique is that it relies on the serendipitous occurrence of an asteroid crossing the same angular coordinates in the sky as those of a star at precisely the right time to observe it. This limits observations to a population on the plane of the ecliptic, makes them hard to predict, difficult to reproduce (though there is the advantage that both ingress and egress of the occultation can be used as independent measurements) and impossible to a priori choose a preferred object. There are relatively few ways to directly measure the angular size of stars and of those methods most are constrained to bright stars to get the necessary signal-to-noise in small $(\sim 1 \mathrm{~m})$ optical telescopes. The VERITAS measurements thus extend the observable phase space to fainter objects that may either be smaller, or further away [26], than those in current catalogues [27]. The fainter in apparent magnitude the stellar population that can be observed then the greater chance probability an observable occultation will take place is enhanced. This could be extremely important in resolving discrepancies between the measured and modelled stellar radii, e.g. in Kstars where the local population brightness distribution maximises at an apparent magnitude of +12 , which in turn are important in calculating the radii of transiting exoplanets. However, to have at least a semblance in target selection at these resolutions requires the use of interferometry another advantage of $\mathrm{TeV}$ telescopes is that they are naturally also consist of arrays of telescopes with large spatial separations. 


\section{Stellar Intensity Interferometry}
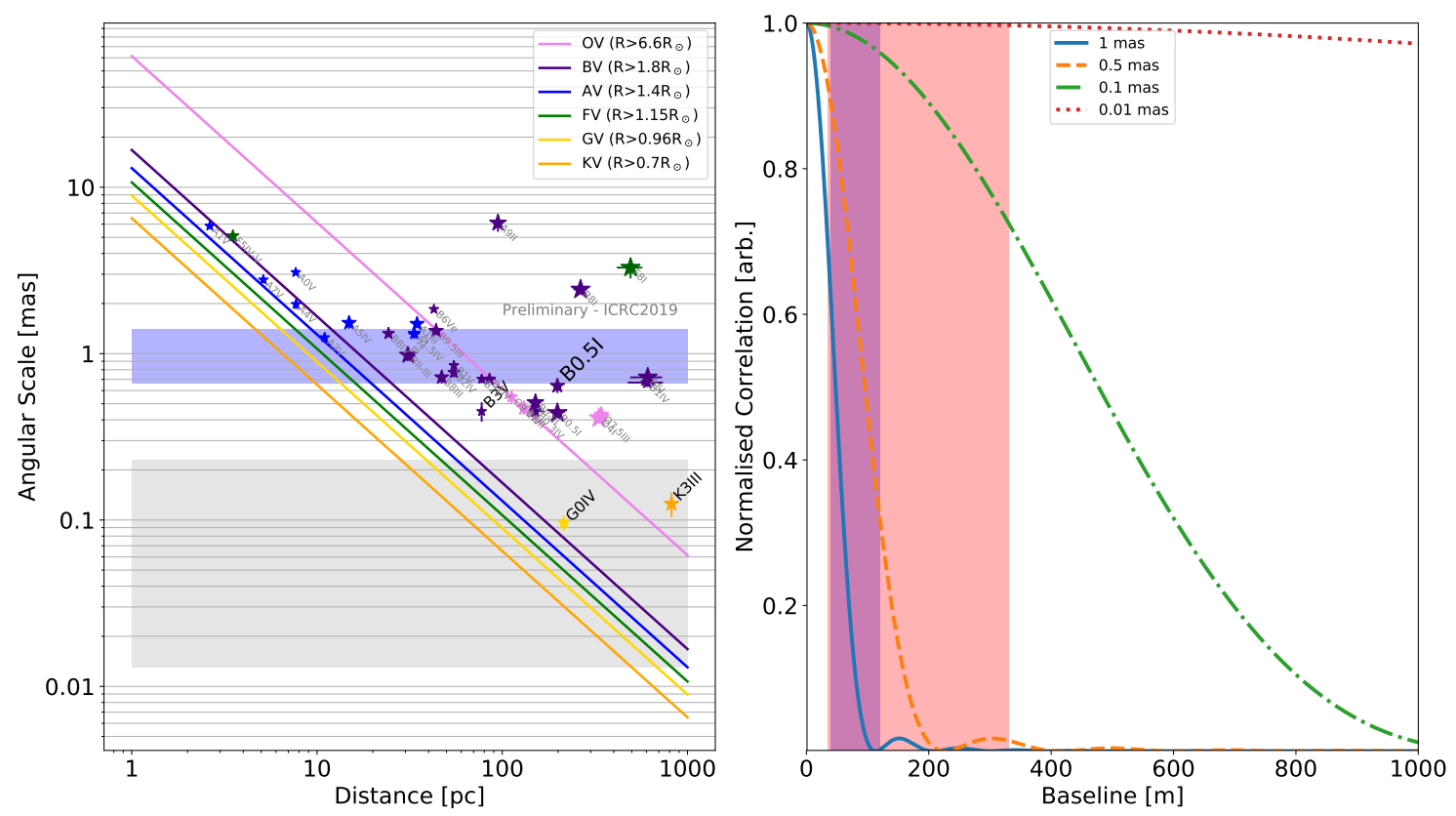

Figure 5: Left: The angular scale as a function of distance for stars directly measured through stellar intensity interferometry or asteroid occultation measurements. The lines are an indication of the expected values for main sequence stars following the Morgan-Keenan classification system on the Hertzprung-Russell diagram. The actual values for the star lines may vary by as much as $20-30 \%$ from the values shown. The blue shaded region shows the angular scales where the first fringe minimises for the telescopes of the VERITAS SII system; the grey shaded region shows the coverage where the Fresnel diffraction deviates from that of a point source for a $12 \mathrm{~m}$ telescope for occultations at the distance of the main belt asteroids. The starred points are direct measurements of the angular diameter of stars from either intensity interferometry [34, 42] or asteroid occultation [10] observations. The colour scale for the stars matches the spectral classification, but not necessarily the luminosity classification for the star. The luminosity classification is indicated by the size of marker from largest for supergiant (I), to smallest for main sequence (V). Right: The expected degree of correlation for intensity fluctuations as a function of telescope separation for a uniform disc approximation of the light intensity for stars of angular diameter 1 mas (solid blue line), 0.5 mas (orange dash), 0.1 mas (green dot-dash), and 0.01 mas (red dotted). The blue shaded region shows the baseline coverage where the first fringe minimises for the telescopes of the VERITAS SII system; the red shaded region shows the baseline coverage for the CHARA phase/amplitude interferometer.

Even stars visible to the naked eye typically have angular diameters on the order of one milliarcsecond (mas) or less, though a handful of red supergiants do extend to a few tens of mas. To reveal details upon stellar surfaces and their environs thus requires imaging with a resolution measured in microarcseconds. Given the diffraction limit of optical light over a telescope aperture this leads to a requirement of kilometre-scale interferometers being necessary to reach such resolutions. The current state of the art is represented by phase/amplitude interferometers such as CHARA in California [28], MROI in New Mexico [29], NPOI in Arizona [30], SUSI in Australia [31], and ESO/VLTI in Chile [32]. This form of interferometer has many operational challenges because 
they require stability of the optical path to within a fraction of an optical wavelength, limiting their operation to the near-infrared and constraining the extension of baselines to no more than a few hundred metres due to the corrections needed to account for atmospheric scintillation. Also, due to the quantum nature of photons, phase/amplitude interferometers are limited in how many optical telescopes can be simultaneously combined; though such limitations do not exist for radio interferometers, enabling large radio arrays with numerous antennas to be built that keep producing spectacular images (e.g. ALMA, EVN, JVLA, LOFAR, MERLIN, NOEMA, VLBA, EHT; SKA in the future).

Intensity interferometry (II) alternatively exploits a second-order effect of light waves, that of the square of the light-wave amplitude, equivalent to its instantaneous intensity. The early development of the technique for astronomical purposes was with an instrument with twin $6.5 \mathrm{~m}$ telescopes made of tessellated mirror panels viewed by photomultiplier tubes (NSII) [33, 34]. If this sounds remarkably similar to a Cherenkov telescope then it should come as little surprise the NSII went on to be used in some of the earliest VHE observations of Cas. A [35]. The baselines for the NSII were adapted by having the telescopes able to move along a $188 \mathrm{~m}$ diameter circular railroad track, but can as easily be done with static telescopes and digital delays in this day and age $[36,37,38,39,40,41,42]$.

\subsection{Principles of Measurement}

The use of the term interferometer is slightly misleading given nothing is actually interfering in the detector, the nomenclature being coined as an analogy to the Michelson interferometer, which has similar scientific goals. Instead the temporal correlations in the arrival times between photons are recorded in two, or more, separated telescopes with the degree of correlation varying as a function of telescope separation. The measurement between a pair of two telescopes is:

$$
\left\langle I_{1}(t) I_{2}(t)\right\rangle=\left\langle I_{1}(t)\right\rangle\left\langle I_{2}(t)\right\rangle\left(1+\left|\gamma_{12}(\mathbf{b})\right|^{2}\right)
$$

where $\gamma_{12}(\mathbf{b})$ is the mutual coherence function of light between locations 1 and 2 separated by baseline $\mathbf{b}$, the quantity commonly measured in amplitude/phase interferometers; \langle\rangle denotes averaging over time. The correlation between non-independently varying intensities $I_{1}$ and $I_{2}$ is 'enhanced' by the coherence parameter. With telescopes close together, the measured fluctuations are essentially simultaneous and thus highly correlated in time, but as the distance increases between telescopes the fluctuations gradually decorrelate and provide a measure of the characteristic angular scale of the source. The mutual coherence as a function of telescope separation for stars of various angular diameters (under the assumption of a uniform disc illumination) is plotted in Figure 3. The larger the star in angular space the shorter the baseline required before the coherence falls away.

Telescopes need only be connected electronically, so the noise budget relates to the relatively long electronic timescales (nanoseconds, thus light-travel distances of tens of centimetres) rather than those of the light wave itself (femtoseconds and nanometres). A realistic time resolution of $3 \mathrm{~ns}$ in the detector chain corresponds to $\sim 1 \mathrm{~m}$ light-travel distance, so that the control of atmospheric path-lengths and telescope imperfections then only needs to correspond to some reasonable fraction of that metre, instead of a fraction of the wavelength of light. The great observational advantage then is that the technique is, to all intents and purposes, essentially insensitive to either 
atmospheric turbulence or telescopic optical imperfections so very long baselines are achievable when observing at short optical wavelengths, even when the optical path may travel through large airmasses far away from the zenith. Given the recorded signals are electronic only, there is the added benefit that these may be freely combined, copied, stored or processed among any number of telescopes, on- or off-line, just as with radio telescopes. However, while intensity interferometry circumvents some of the major limitations of phase interferometry, it does not do so without cost, and it instead comes with observational challenges of its own. Averaging the intensity fluctuations in the photon stream over a realistic time resolution of a few nanoseconds is much longer than the coherence time scales over which the quantum intensity fluctuations are fully developed (pico- or femtoseconds) so precise measurements with good photon statistics are required for the correlation measurement. Intensity interferometry must therefore be carried out with a set of large telescopes, on the order of at least 5-10 metres in aperture, which is the main reason why this method has not been actively pursued until recently in optical astronomy after the NSII reached its sensitivity limits. For image reconstruction, a greater complication comes from the correlation function for the electric field, $\gamma_{12}$, not being directly measured, but only the square of its modulus, $\left|\gamma_{12}\right|^{2}$. Since this does not preserve phase information, a simple inverse Fourier transform does not suffice to obtain full two-dimensional images. Thankfully methods have been developed in various disciplines (e.g., coherent diffraction imaging in X-rays), demonstrating how rather complex images can also be reconstructed through phase retrieval algorithms or correlations between more than two telescopes [43]. To enable true two-dimensional imaging, a multi-telescope grid is therefore required to provide numerous baselines, and cover the interferometric Fourier-transform $(u, v)$-plane, analogous to radio arrays.

\subsection{The sensitivity limits of an intensity interferometer}

To be able to define a realistic observing programme inherently requires an understanding of the noise properties of the measurement of the second-order coherence of light, which differs from standard optical observations. For one pair of telescopes, the signal-to-noise ratio for polarized light is given in a first approximation by:

$$
(S / N)_{\mathrm{RMS}}=A \alpha \eta\left|\gamma_{12}(\mathbf{b})\right|^{2} \sqrt{\Delta f T / 2}
$$

where $A=\sqrt{A_{1} A_{2}}$ is the geometric mean of the areas of the two telescopes; $\alpha$ is the quantum efficiency of the optics plus detector system; $\eta$ is the flux of the source in photons per unit optical bandwidth, per unit area, and per unit time; $\Delta f$ is the electronic bandwidth of the detector plus signal-handling system, and $T$ is the integration time. The signal-to-noise ratio naturally depends on a number of readily understandable parameters such as telescope size, detector efficiency, time resolution and total observing time, but its success also fundamentally depends on source inherent properties, like temperature, to generate a sufficiently intense photon field to have the correlated intensity fluctuations in the first place. The NSII covered bright (down to second-magnitude) and relatively hot stars $(\geq 9000 \mathrm{~K})$ in its catalogue, see figure 3 (left), the estimates of limiting magnitude for modern electronics place the limiting visual magnitude closer to $\sim 6$ th magnitude for a $12 \mathrm{~m}$ class telescope [44] for stars of spectral type hotter than G. Plotted in Figure 6 is the observing time needed to reach a $\mathrm{S} / \mathrm{N}=3$ for $\gamma_{12}=0.2$ as a function of stellar magnitude, telescope aperture 
and electronics bandwidth for a number of telescope types. The larger the telescope the quicker it can reach the required signal-to-noise level and the fainter a source it can detect, but even a $4 \mathrm{~m}$ diameter aperture telescope can exceed the sensitivity of the NSII with the use of modern higher quantum efficiency photodetectors and higher bandwidth electronics.

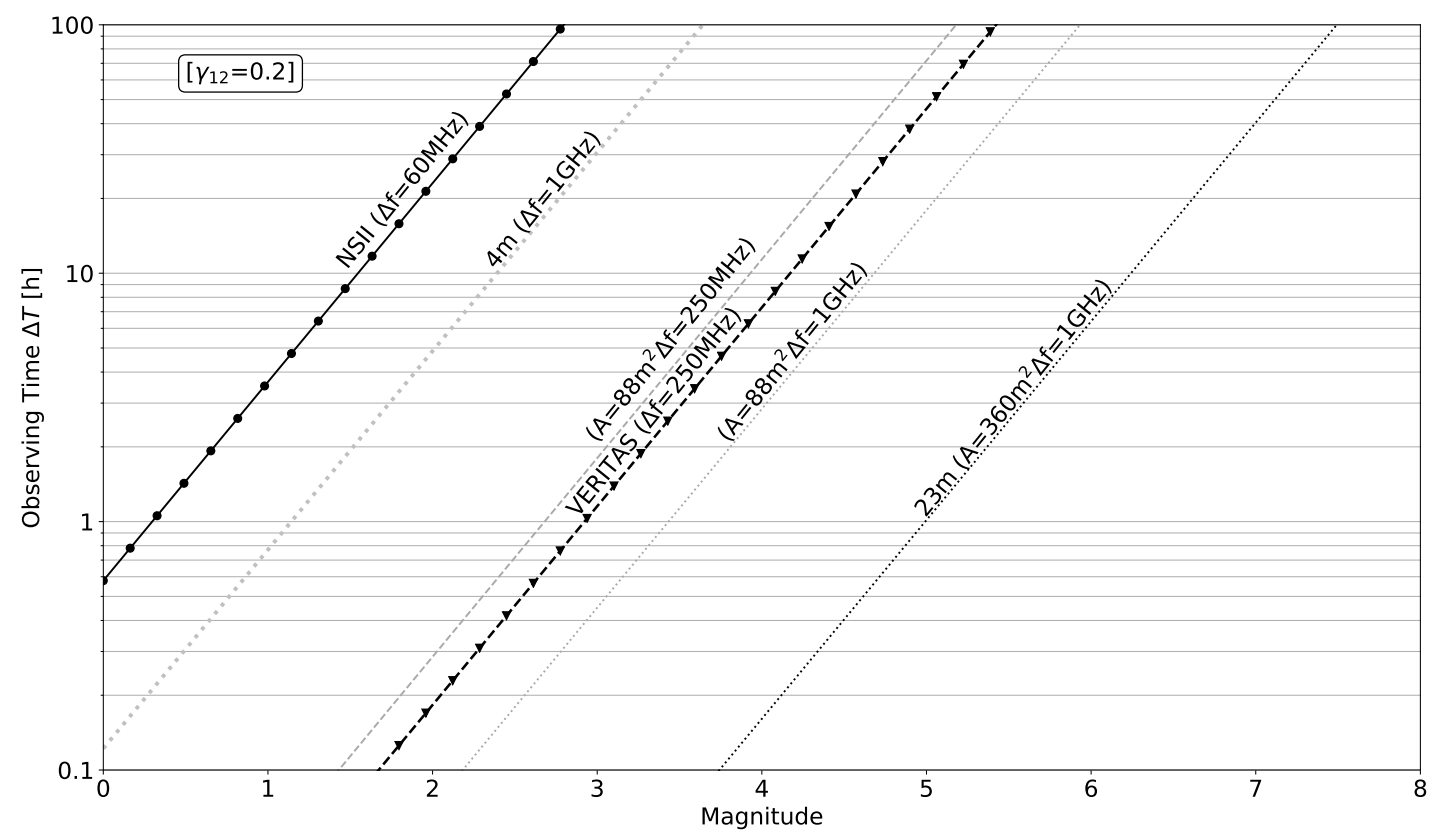

Figure 6: The observing time needed to reach a $\mathrm{S} / \mathrm{N}=3$ for a coherence function of light $\gamma_{12}=0.2$ as a function of magnitude for telescopes of varying mirror area, including those of relevance to the future CTA[52] telescopes, and electronics bandwidth. The NSII is depicted by the solid line and round points, the VERITAS SII system by the triangle points. An electronics bandwidth of $250 \mathrm{MHz}$ and $1 \mathrm{GHz}$ by a dashed line and dotted line respectively.

\section{Free Space Optical Communication and the Optical Search for Extra Terrestrial Intelligence}

The use of radio waves to transmit information from spacecraft to Earth is a bottleneck in terms of communications and the increased bandwidth available in using lasers is a tempting alternative when telescopes of sufficiently large aperture to allow optical communications are available [45]. Indeed such an ability to transmit significant amounts of data across even interstellar distances was realised very early on in the developmental lifetime of the laser [46]. As shown in Figure 4 any design of Cherenkov telescope will have sensitivity down to the single photon counting per square-centimetre level, which enables them push the search horizon far beyond many of the dedicated (small) search instruments. Add to that the large field of view which means searches can be conducted of multiple star systems, and simultaneously to norm $\mathrm{TeV}$ data acquisition, making for an extremely inexpensive survey programme. Details on some of the existing upper limits can be found in $[47,48,49]$. 


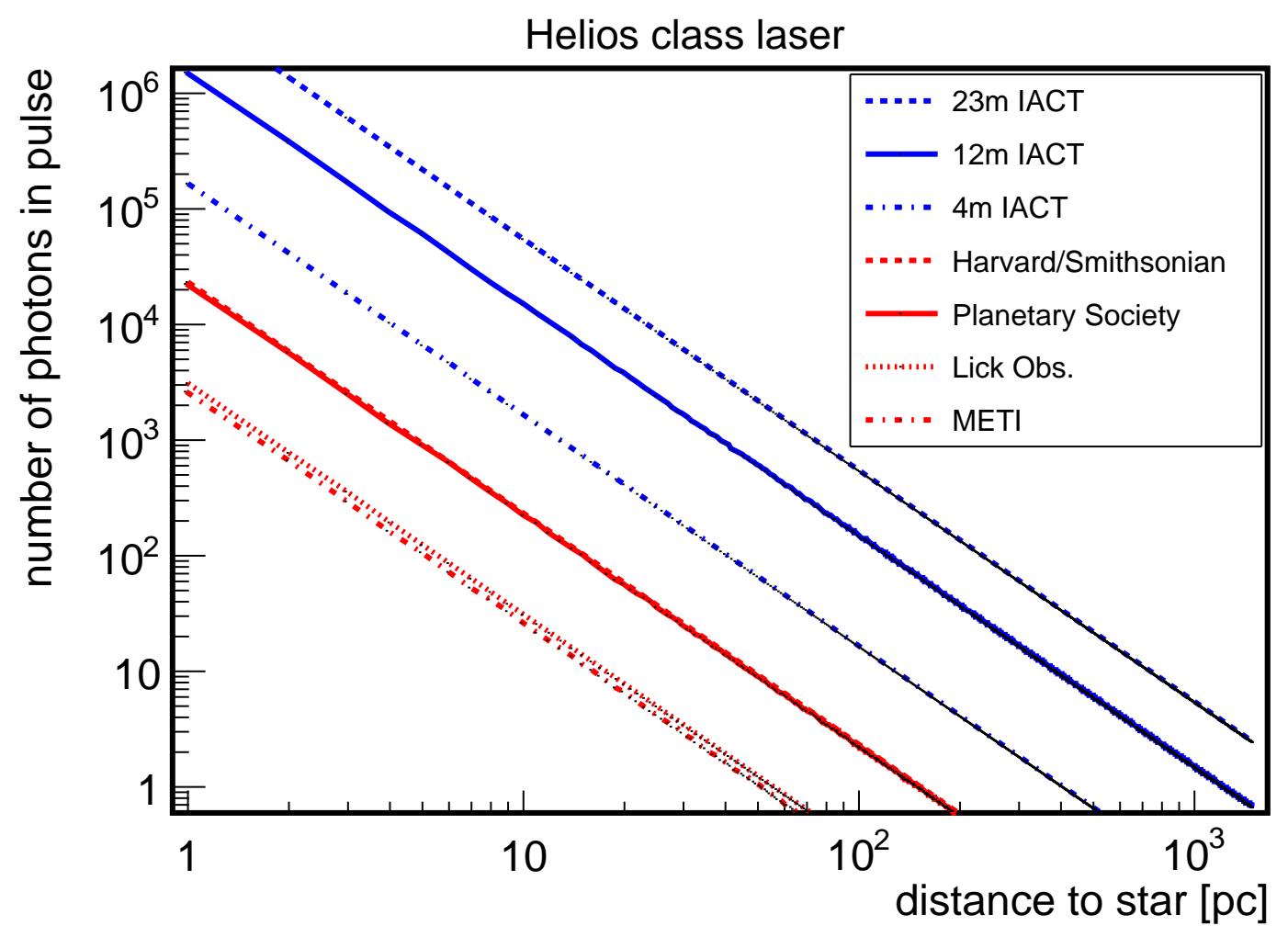

Figure 7: The number of photons that can be detected from a Helios class laser pulse as a function of distance for telescopes of various apertures.

\section{Discussion}

In Figure 5 is plotted the angular resolution as a function of wavelength for a number of facilities. To date the highest angular resolutions in astronomical observations have been achieved by continental scale radio and sub-mm facilities so it is remarkable to think that a single Cherenkov telescope facility can match those angular resolutions in the optical. The stellar angular diameter measurements already match the larger end of the angular resolution for the Event Horizon Telescope that provided the first images of the shadow for the supermassive black hole at the centre of M 87 [50]. Of course, the asteroid occultation observations only provide information on the 1 dimensional angular diameter for a star, but that is crucial information for both understanding stellar modelling and also in determining the radii of transiting exoplanets. Of the few methods available to determine stellar angular diameters independent of modelling, it is from asteroid occultation observations with Cherenkov telescopes that we have the best opportunity to resolve the growing discrepancy between measured and modelled K star diameters [26], of especial interest in the hunt for terrestrial-like exoplanets in habitable zones. To get an effective observation programme going for more than a handful of detections requires sensitivity to the level of 13th magnitude and fainter stars, the fact that the Crab optical pulsar at 16th magnitude has been so comprehensively studied gives confidence that such an instrumental sensitivity level is within reach by all currently operating IACTs. The occultation technique is more traditionally used in determining parameters of the 


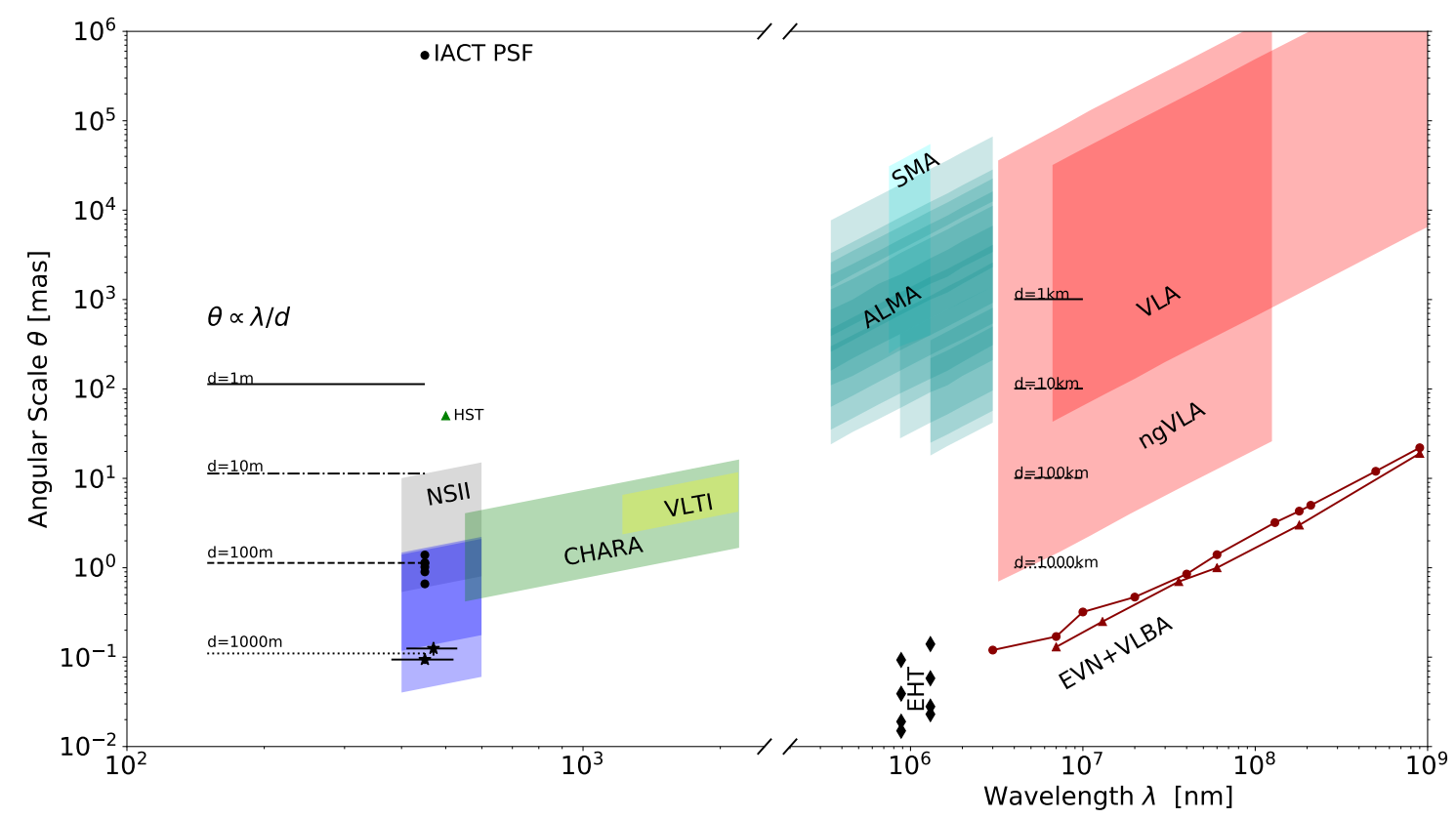

Figure 8: The angular resolution reached as a function of wavelength for a number of facilities. The dots indicate the instantaneous baseline coverage of the VERITAS SII system pointing at zenith, the blue shaded regions show the phase space that could be covered by a comparable CTA SII system. The stars are the stellar diameter measurements made from asteroid occultation observations made by VERITAS [10].

occulting object and here the light collecting and scintillation noise reduction power of $\mathrm{TeV}$ telescopes could provide useful also in the hunt for the unidentified population of sub-km Kuiper Belt Objects (KBOs) [51] that reveal information on the planetary formation history of the solar system. These KBOs are too small and distant to be imaged by optical telescopes, in the way that asteroids and large KBOs are found and tracked and this is another aspect of $\mathrm{TeV}$ telescopes that comes into play in such an area of investigation. Because the $\mathrm{KBO}$ is so small and can not be imaged it would be impossible to identify it conclusively by occultation alone as its distance would be ambiguous, however, by using different optical bandpass filters on the pixels in an array of telescopes the distance to the occulting object can be determined from the wavelength dependent differences in the arrival of the diffraction fringes.

The main drawback of the occultation method is that it relies on the serendipitous overlapping of two objects on the celestial sphere such that the shadow cast passes over a specific fixed telescope site, during the night time, with clear skies overhead. Interferometry is another of the techniques for directly measuring stellar radii, but with the greater freedom of greater selection in which targets to observe and when they can be observed. Intensity interferometry enables measurements in the blue end of the spectrum and so at different optical depths to complement and extend the measurements made by phase/amplitude interferometers, and the km-scale baselines that a CTA SII system $[52,53]$ could leverage means that it would match the sub-mas angular scale regime of asteroid occultation diffraction fringes. Limb darkening in the stellar light profile is another complication in determining exoplanet radii and having a sensitive instrument able to measure 
the higher order fringes in the 1-D correlation plot enables the amount of limb darkening to be determined in a manner that breaks the degeneracy in a sea of limb darkening prescriptions. But measuring stellar angular diameters is perhaps the least of the science that can be performed with an intensity interferometer $[54,55,53]$. On the one hand SII is limited to much brighter stars than can be observed with the occultation method, on the other it is the 2 dimensional imaging potential from the vast number of baselines afforded by a large array that is the most exciting prospect. From the distorted shapes of rapidly rotating stars, to star-spots and other surface features revealing the underlying physics processes at work in these massive and complex systems. The sheer amount of coverage of the $(\mathrm{u}, \mathrm{v})$ imaging plane, even after having to account for phase retrieval methods, would enable imaging at an unprecedented scale in the optical.

Nor should it be thought that high time resolution optical astronomy with $\mathrm{TeV}$ telescopes is limited to specialised high angular resolution science cases. There are many types of observations that also benefit from the wide field of view cameras and can even be performed simultaneously to the normal $\mathrm{TeV}$ observations, such as the aforementioned OSETI or the search for counterparts to Fast Radio Bursts $[57,56]$. When it comes to prospects for Non-gamma-ray applications of $\mathrm{TeV}$ telescopes the future seems optically bright indeed.

\section{Acknowledgments}

In gathering material for these proceedings, the author wishes to acknowledge many fruitful discussions with Colin Carlile, Dainis Dravins, Tarek Hassan, Jamie Holder, Dave Kieda, Stephan Le Bohec, Nolan Matthews, and the many members of the CTA Intensity Interferometry Science Working Group. This research is supported by grants from the U.S. Department of Energy Office of Science, the U.S. National Science Foundation and the Smithsonian Institution, and by NSERC in Canada. We acknowledge the excellent work of the technical support staff at the Fred Lawrence Whipple Observatory and at the collaborating institutions in the construction and operation of the VERITAS instrument that provided many of the results presented here.

\section{References}

[1] CMG Lee. https://commons.wikimedia.org/wiki/File: Comparison_optical_telescope_primary_mirrors.svg. CC-BY-SA-3.0

[2] B. Lacki, Cherenkov telescopes as optical telescopes for bright sources: today's specialized 30-m telescopes?, MNRAS 416, 3075 (2011). [arXiv:1106.3093]

[3] J. Osborn et al. Atmospheric scintillation in astronomical photometry, MNRAS 452, 1707 (2015). [arXiv:1506.06921]

[4] A. T. Young Photometric error analysis. VIII. The temporal power spectrum of scintillation, AO 8, 869 (1969).

[5] D. Dravins et al., Atmospheric Intensity Scintillation of Stars. III. Effects for Different Telescope Apertures, PASP 110, 747 (1998).

[6] A. F. Cook et al., Flux of optical meteors down to $M_{P G}=+12, \operatorname{MNRAS~193,~} 645$ (1980).

[7] A. De Rújula \& S. L. Glashow Nuclearites - a novel form of cosmic radiation, Nature 312, 734 (1984). 
[8] N. Porter et al. A search for evidence of nuclearites in astrophysical pulse experiments, Nature 316, 49 (1985).

[9] T. Nagai et al., Focal Plane Instrumentation of VERITAS in proceedings of the 30th ICRC 1437 (2007). [arXiv:0709.4517]

[10] W. Benbow et al. Direct measurement of stellar angular diameters by the VERITAS Cherenkov telescopes, Nature Astronomy 3, 511 (2019). [arXiv:1904.06324]

[11] F. Lucarelli et al., The central pixel of the MAGIC telescope for optical observations, NIMA 589, 415 (2008).

[12] C. Deil et al. Capability of Cherenkov telescopes to observe ultra-fast optical flares, APh 31, 156 (2009). [arXiv:0812.3966]

[13] S. Griffin, Searching for Fast Optical Transients using VERITAS Cherenkov Telescopes in roceedings of 32nd ICRC 9, 38 (2011).

[14] R. Srinivasan et al., Detection of the optical Crab pulsar with an atmospheric Cerenkov telescope in proceedings of Towards a Major Čerenkov Detector, 51 (1997).

[15] R. Lessard et al., Search for Pulsed TEV Gamma-Ray Emission from the Crab Pulsar, ApJ 531, 942 (2000). [astro-ph/9912520]

[16] M. de Naurois et al. Measurement of the Crab Flux above $60 \mathrm{GeV}$ with the CELESTE Cerenkov Telescope, ApJ 566, 343 (2002). [astro-ph/0107301]

[17] E. Oña-Wilhelmi et al., Determination of the night sky background around the Crab pulsar using its optical pulsation, APh 22, 95 (2004). [astro-ph/0405088]

[18] Fortin, P. PhD thesis (McGill) (2005).

[19] J. Hinton et al. Precision measurement of optical pulsation using a Cherenkov telescope, APh 26, 22 (2006).

[20] J. Albert et al. VHE $\gamma$-Ray Observation of the Crab Nebula and its Pulsar with the MAGIC Telescope, ApJ 674, 1037 (2008). [arXiv:0705.3244]

[21] O. Celik, Observations of the Crab Nebula and Pulsar with VERITAS in Proceedings of 30th ICRC 2, 847 (2008).

[22] C. L. Morbey, Distortion mechanisms for lunar occultation diffraction patterns, AJ 79, 1304 (1974).

[23] S. T. Ridgway, Considerations for the application of the lunar occultation technique, AJ 82, 511 (1977).

[24] F. Roques, M. Moncuquet \& B. Sicardy, AJ 93, 1549 (1987).

[25] G. Pojmanski, 1997, The All Sky Automated Survey, Acta Astronomica 47, 467 (1997).

[26] T. Hassan et al., Proving the outstanding capabilities of Imaging Atmospheric Cherenkov Telescopes in high time resolution optical astronomy, these proceedings

[27] G. Duvert, VizieR Online Data Catalog, 2345 (2016).

[28] T. A. ten Brummelaar et al. First Results from the CHARA Array. II. A Description of the Instrument, ApJ 628, 453 (2005).

[29] D. F. Buscher et al. The Conceptual Design of the Magdalena Ridge Observatory Interferometer, JAI 2, 1340001 (2013). [arXiv:1307.0391] 
[30] J. T. Armstrong et al. The Navy Prototype Optical Interferometer, ApJ 496, 550 (1998).

[31] J. Davis et al. The Sydney University Stellar Interferometer: A Major Upgrade to Spectral Coverage and Performance, PASA 24, 138 (2007).

[32] Glindemann et al. The VLT Interferometer: a unique instrument for high-resolution astronomy in Proceedings of SPIE 4006, 2 (2000).

[33] R. Hanbury Brown, Stellar Interferometer at Narrabri Observatory, Nature 218, 637 (1968).

[34] R. Hanbury Brown, The Angular Diameters of 32 Stars, MNRAS 167, 121 (1974).

[35] J. E. Grindlay et al., Evidence for the detection of gamma rays from Centaurus A at $E_{\gamma} \geq 3 \times 10^{11} \mathrm{eV}$, ApJ 197, 9 (1975).

[36] E. P. Horch et al., SCSI: the Southern Connecticut Stellar Interferometer in proceedings of SPIE 9907, 99071 (2016).

[37] L. Zampieri et al., Intensity interferometry with Aqueye+ and Iqueye in Asiago in proceedings of SPIE 9907, 99070 (2016).

[38] G. Pilyavsky et al., Single-Photon Intensity Interferometry (SPIIFy): utilizing available telescopes, MNRAS 467, 3048 (2017).

[39] P. K. Tan \& C. Kurtsiefer, Temporal intensity interferometry for characterization of very narrow spectral lines, MNRAS 469, 1617 (2017).

[40] W. Guerin et al., Temporal intensity interferometry: photon bunching in three bright stars, MNRAS 472, 4126 (2017). [arXiv:1708.06119]

[41] W. Guerin et al., Spatial intensity interferometry on three bright stars, MNRAS 480, 245 (2018). [arXiv:1805.06653]

[42] N. Matthews et al., Astrophysical measurements with the VERITAS Stellar Intensity Interferometer, these proceedings

[43] P. D. Nuñez et al., Imaging submilliarcsecond stellar features with intensity interferometry using air Cherenkov telescope arrays, MNRAS 424, 1006 (2012). [arXiv:1205.5743]

[44] S. Le Bohec \& J. Holder, Optical Intensity Interferometry with Atmospheric Cerenkov Telescope Arrays, ApJ 649, 399 (2006). [astro-ph/0608305]

[45] A. Carrasco-Casado et al., Feasibility of utilizing Cherenkov Telescope Array gamma-ray telescopes as free-space optical communication ground stations, AO 52, 2353 (2013).

[46] R. N. Schwartz \& C. H. Townes, Interstellar and Interplanetary Communication by Optical Masers, Nature, 190, 205 (1961).

[47] J. Holder et al., Optical SETI with Imaging Cherenkov Telescopes in proceedings of the 29th ICRC 5, 387 (2005). [astro-ph/0506758]

[48] D. Hanna et al., OSETI with STACEE: A Search for Nanosecond Optical Transients from Nearby Stars, AsBio 9, 345 (2009). [arXiv:0904.2230]

[49] A. U. Abeysekara et al., A Search for Brief Optical Flashes Associated with the SETI Target KIC 8462852, ApJL 818, L33 (2016).

[50] Event Horizon Telescope Collaboration, First M87 Event Horizon Telescope Results. I. The Shadow of the Supermassive Black Hole, ApJ Letters 875, 1 (2019). [arXiv:1906.11238] 
[51] B. Lacki, On the use of Cherenkov Telescopes for outer Solar system body occultations, MNRAS 445, 1858 (2014). [arXiv:1402.1179]

[52] Acharya et al., Introducing the CTA concept, APh 43, 3 (2013).

[53] The CTA Consortium, Science with Cherenkov Telescope Array, World Scientific Publishing Co. Pte Ltd doi : $10.1142 / 10986$ (2019). [arXiv:1709.07997]

[54] D. Dravins et al., Stellar intensity interferometry: Prospects for sub-milliarcsecond optical imaging, New Astronomy Reviews 56, 143 (2012). [arXiv:1207.0808]

[55] D. Dravins et al., Optical intensity interferometry with the Cherenkov Telescope Array, APh 43, 331 (2013). [arXiv:1204.3624]

[56] V. Acciari et al., Constraining very-high-energy and optical emission from FRB 121102 with the MAGIC telescopes, MNRAS 481, 2479 (2018). [arXiv:1809.00663]

[57] J. Holder et al., VERITAS Observations of Fast Radio Bursts, these proceedings 